\title{
Electrical conductivity and thermal dilepton rate from quenched lattice QCD
}

\author{
O. Kaczmarek and A. Francis \\ Fakultät für Physik, Universität Bielefeld, D-33615 Bielefeld, Germany \\ E-mail: okacz@physik.uni-bielefeld.de
}

\begin{abstract}
We report on a continuum extrapolation of the vector current correlation function for light valence quarks in the deconfined phase of quenched QCD. This is achieved by performing a systematic analysis of the influence of cut-off effects on light quark meson correlators at $T \simeq 1.45 T_{c}$ using clover improved Wilson fermions [1]. We discuss resulting constraints on the electrical conductivity and the thermal dilepton rate in a quark gluon plasma. In addition new results at 1.2 and $3.0 T_{c}$ will be presented.
\end{abstract}

\section{Introduction}

The measured dilepton rates in heavy ion experiments show an enhancement in the low invariant mass regime of a few hundred $\mathrm{MeV}$ and are getting sensitive to transport properties which are relevant in the hydrodynamic regime of small invariant masses [2, 3]. The spectral representation of the correlation functions of the vector current directly relates to the invariant mass spectrum of dileptons and photons and in the limit of small frequencies determines a transport coefficient, in the case of the vector correlation function of light quarks, the electical conductivity.

At temperatures relevant for current heavy ion experiments, non-perturbative techniques are mandatory for the determination of those quantities. Perturbative studies of the vector spectral functions [4, 5] and also the inclusion of nonperturbative aspects through the hard thermal loop resummation scheme [6] break down, especially in the low invariant mass region indicated by an infrared divergent Euclidean correlator [7] leading to an infinite electrical conductivity. Instead it was demonstrated that the spectral function at low invariant masses will increase linearly resulting in a finite electrical conductivity of the quark gluon plasma [8, 9].

In [1] we have analyzed the behaviour of the vector correlation function at $T \simeq 1.45 T_{c}$ and performed its extrapolation to the continuum limit based on precise data at various lattice sizes, corresponding to different lattice cutoffs. While only small finite volume effects were observed in this study, large cutoff effects in the correlation functions require small lattice spacings and a proper continuum extrapolation to obtain reliable results for the determination of the spectral properties and the extraction of the dilepton rates and transport coefficients. 

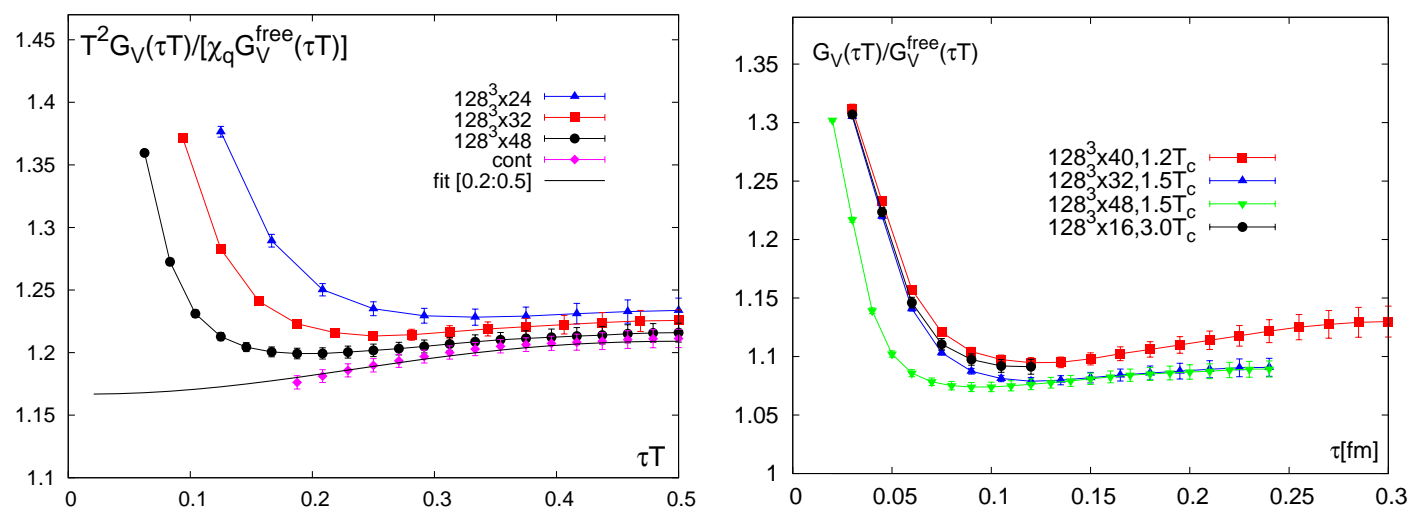

Figure 1. The vector correlation function $G_{V}(\tau T)$ calculated at $T \simeq 1.45 T_{c}$ (left) normalized by the continuum version of the corresponding free vector correlation function together with the continuum extrapolation [1]. The same quantity on the finest lattices for three different temperatures (right).

\section{Thermal vector correlation and spectral function}

In fig. 1 (left) our results for the vector correlation function normalized by the corresponding continuum free correlator and the quark number susceptibilty $\chi_{q}$ are shown for various lattice sizes $N_{\sigma}^{3} \times N_{\tau}$ together with the continuum extrapolation [1]. The strong cutoff effects at small separations $\tau T$ on the lattices with small temporal extend $N_{\tau}$ clearly show the necessity for the continuum extrapolation. Only on the finest lattice and in the extrapolation the relevant physical behaviour of the vector correlation function becomes apparent and a reliable continuum extrapolation at distance $\tau T \gtrsim 0.2$ could be performed.

We used an Ansatz for the vector spectral function,

$$
\begin{aligned}
& \rho_{00}(\omega)=-2 \pi \chi_{q} \omega \delta(\omega), \\
& \rho_{i i}(\omega)=2 \chi_{q} c_{B W} \frac{\omega \Gamma / 2}{\omega^{2}+(\Gamma / 2)^{2}}+\frac{3}{2 \pi}(1+k) \omega^{2} \tanh (\omega / 4 T),
\end{aligned}
$$

that depends on four temperature dependent parameters; the quark number susceptibility $\chi_{q}(T)$, the strength $\left(c_{B W}(T)\right)$ and width $(\Gamma(T))$ of the Breit-Wigner peak and the parameter $k(T)$ that parametrizes deviations from a free spectral function at large energies. At high temperature and for large energies, $\omega / T \gg 1$, we expect to find $k(T) \simeq \alpha_{s} / \pi$.

The parameters are determined by a fit to the continuum extrapolated vector correlation function. In order to analyze the influence of the low energy structure of the spectral function and to analyze the systematic uncertainties of the Ansatz, we have smoothly truncated the continuum contribution at some energy $\omega_{0}$ by multiplying the second term in (2) with $\Theta\left(\omega_{0}, \Delta_{\omega}\right)=\left(1+\mathrm{e}^{\left(\omega_{0}^{2}-\omega^{2}\right) / \omega \Delta_{\omega}}\right)^{-1}$. Details of the fit procedure including additional information obtained from thermal moments of the spectral functions are discussed in [1]. The resulting spectral functions are shown in Fig. 2] for different values 

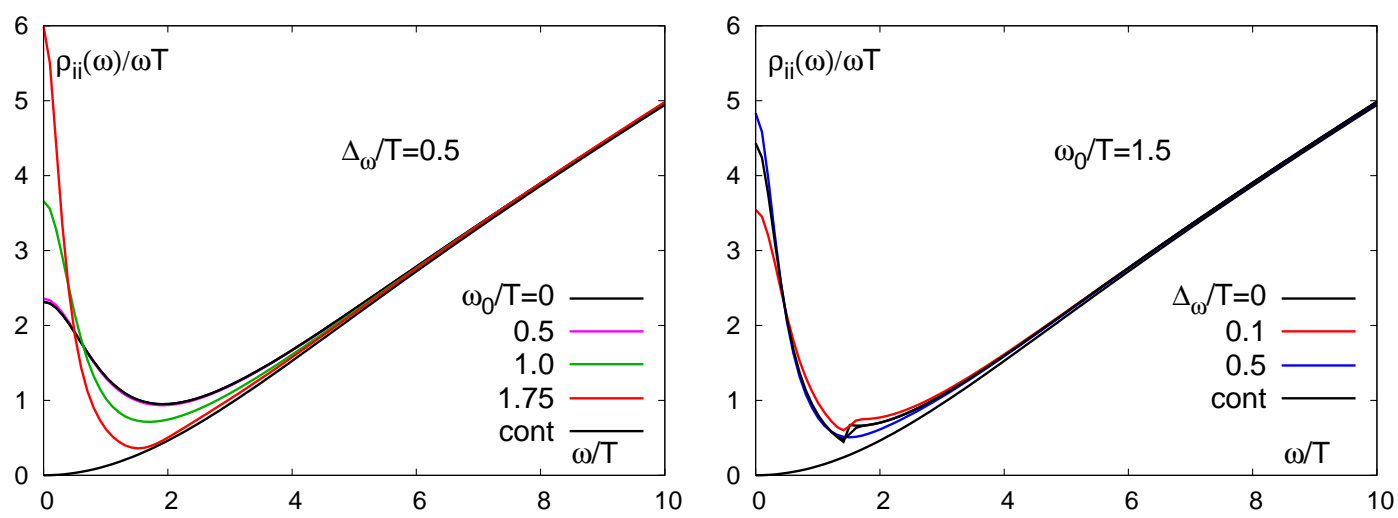

Figure 2. Spectral functions obtained from fits to the vector correlation function 1 . For comparison we also show only the continuum part of the spectral function. The left hand figure shows results for different values of the cut-off $\left(\omega_{0}\right)$ and fixed width $\left(\Delta_{\omega}\right)$. The right hand figure shows results for fixed $\omega_{0} / T=1.5$ and several values of $\Delta_{\omega}$. The curve labeled 'cont' is the continuum contribution to the fit described in (2).

of $\omega_{0}$ and $\Delta_{\omega}$ that lead to $\chi^{2} /$ d.o.f smaller than unity.

\section{Electrical conductivity and thermal dilepton rate}

In Fig. 3 we show the thermal dilepton rate

$$
\frac{\mathrm{d} N_{l^{+} l^{-}}}{\mathrm{d} \omega \mathrm{d}^{3} p}=C_{e m} \frac{\alpha_{e m}^{2}}{6 \pi^{3}} \frac{\rho_{V}(\omega, \vec{p}, T)}{\left(\omega^{2}-\vec{p}^{2}\right)\left(\mathrm{e}^{\omega / T}-1\right)},
$$

for two massless $(u, d)$ flavors. The results are compared to a dilepton spectrum calculated within the hard thermal loop approximation [6] using a thermal quark mass $m_{T} / T=1$. For $\omega / T \geq 2$ the results are in good agreement and for $1 \lesssim \omega / T \lesssim 2$ differences between the HTL spectral function and our numerical results are about a factor two, which is the intrinsic uncertainty in our spectral analysis.

While for energies $\omega / T \lesssim 1$ the HTL results grow too rapidly, from our numerical results we obtain a finite electrical conductivity,

$$
1 / 3 \lesssim \frac{1}{C_{e m}} \frac{\sigma}{T} \lesssim 1 \text { at } T \simeq 1.45 T_{c}
$$

\section{Conclusions}

We have performed a detailed analysis of the vector correlation function at a fixed temperature of $1.45 T_{c}$ in the high temperature phase of quenched QCD. The results allowed for a determination of the spectral properties and the resulting dilepton rate as well as an estimate for the electrical conductivity of the QGP.

First results at different temperatures, 1.2 and $3.0 T_{c}$ (Fig. 1(right)), show a qualitatively similar behaviour for the vector correlation function. Even at the smallest temperature 

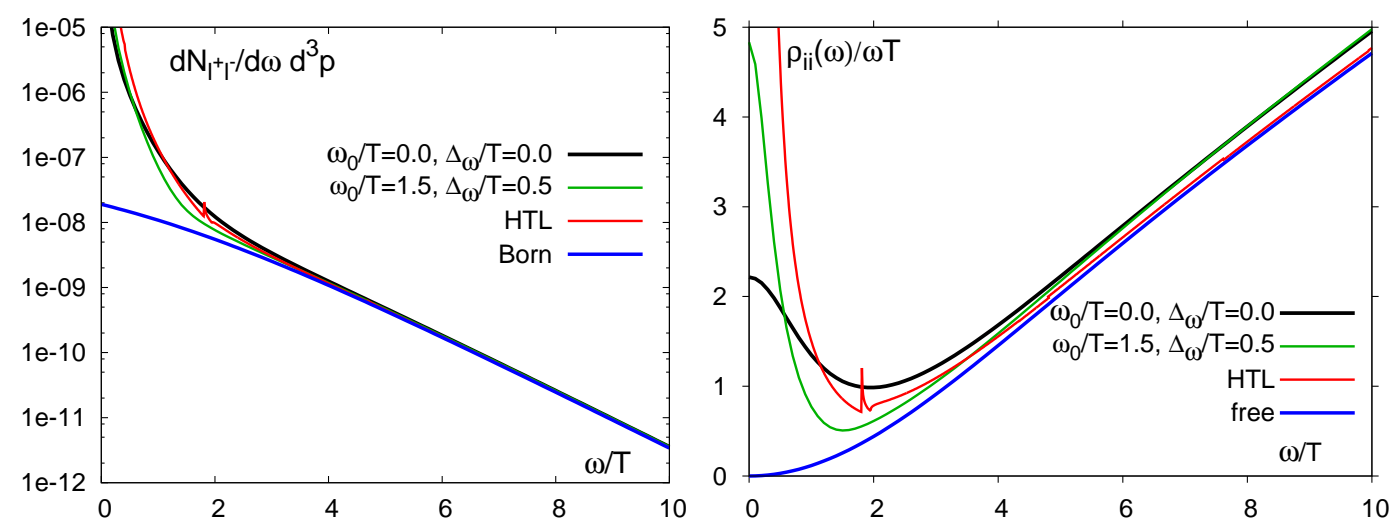

Figure 3. Thermal dilepton rate in 2-flavor QCD (left). Shown are results from fits without a cut-off on the continuum contribution $\left(\omega_{0} / T=0\right)$ and with the largest cutoff tolerable in our fit ansatz $\left(\omega_{0} / T=1.5\right)$. The HTL curve is for a thermal quark mass $m_{T} / T=1$ and the Born rate is obtained by using the free spectral function. The right hand part of the figure shows the spectral functions that entered the calculation of the dilepton rate.

of $1.2 T_{c}$ no signals for a sizeable contribution of a $\rho$-resonance are visible. However a detailed analysis of the spectral properties at temperatures close to the critical one remains to be performed in future.

Furthermore in order to analyze to what extent the low mass enhancement observed in our estimate for the dilepton rate can account for the experimentally observed enhancement of dilepton rates at low energies [2, 3, 10] results over the whole temperature region probed experimentally as well as knowledge on its momentum dependence is needed together with a realistic model for the hydrodynamic expansion of dense matter created in heavy ion collisions [11].

\section{References}

[1] H. T. Ding et al., Phys. Rev. D 83, 034504 (2011).

[2] A. Adare et al. (PHENIX Collaboration), Phys. Rev. Lett. 104, 132301 (2010) and Phys. Rev. C 81, 034911 (2010).

[3] J. Zhao (for the STAR collaboration), these proceedings, arXiv:1106.6146.

[4] for an overview and further references, see for instance: F. Gelis, Nucl. Phys. A 715, 329 (2003).

[5] J. P. Blaizot and F. Gelis, Eur. Phys. J. C 43, 375 (2005).

[6] E. Braaten and R.D. Pisarski, Nucl. Phys. B 337, 569 (1990).

[7] F. Karsch, M. G. Mustafa and M. H. Thoma, Phys. Lett. B 497, 249 (2001).

[8] P. B. Arnold, G. D. Moore and L. G. Yaffe, JHEP 0011, 001 (2000); JHEP 0112, 009 (2001) and JHEP 0305, 051 (2003).

[9] P. Aurenche, F. Gelis, G. D. Moore and H. Zaraket, JHEP 0212, 006 (2002).

[10] D. Adamova et al., Phys. Lett. B 666, 425 (2008).

[11] H. van Hees and R. Rapp, Nucl. Phys. A 806, 339 (2008). 